LAWRENCE LIVERMORE N A T IO N A L LABORATORY
Speckle Imaging of Titan at 2 microns: Surface Albedo,Haze Optical Depth, and Tropospheric Clouds 1996-1998

S. G. Gibbard, B. A. Macintosh, D. Gavel, C. E. Max, I. de Pater, H. G. Roe, A. M. Ghez, E. F. Young, C. P. McKay

February 27, 2004

Icarus 
This document was prepared as an account of work sponsored by an agency of the United States Government. Neither the United States Government nor the University of California nor any of their employees, makes any warranty, express or implied, or assumes any legal liability or responsibility for the accuracy, completeness, or usefulness of any information, apparatus, product, or process disclosed, or represents that its use would not infringe privately owned rights. Reference herein to any specific commercial product, process, or service by trade name, trademark, manufacturer, or otherwise, does not necessarily constitute or imply its endorsement, recommendation, or favoring by the United States Government or the University of California. The views and opinions of authors expressed herein do not necessarily state or reflect those of the United States Government or the University of California, and shall not be used for advertising or product endorsement purposes. 


\title{
SPECKLE IMAGING OF TITAN AT 2 MICRONS: SURFACE ALBEDO, HAZE OPTICAL DEPTH, AND TROPOSPHERIC CLOUDS 1996-1998
}

\author{
S.G. Gibbard ${ }^{1}$, B. Macintosh ${ }^{1}$, D. Gavel ${ }^{1,2}$, C.E. $\operatorname{Max}^{1,2}$, \\ I. de Pater ${ }^{3}$, H. G. Roe ${ }^{3,4}$, A.M. Ghez ${ }^{5}$, \\ E.F. Young ${ }^{6}$ and C.P. McKay ${ }^{7}$ \\ ${ }^{1}$ Institute of Geophysics and Planetary Physics \\ Lawrence Livermore National Laboratory \\ Livermore, CA 94550 USA; sgibbard@igpp.ucllnl.org \\ ${ }^{2}$ University of California at Santa Cruz \\ ${ }^{3}$ Dept. of Astronomy, University of California at Berkeley \\ ${ }^{4}$ Division of Geological and Planetary Sciences \\ California Institute of Technology \\ ${ }^{5}$ Dept. of Physics, University of California at Los Angeles \\ ${ }^{6}$ Southwest Research Institute, Boulder, Colorado \\ ${ }^{7}$ NASA Ames Research Center, Moffett Field, California
}

Proposed running head: Titan: 2 micron speckle observations

keywords: Titan; infrared observations; surfaces,satellite

Number of pages: 35

Number of tables: 3

Number of figures: 6 


\begin{abstract}
We present results from 14 nights of observations of Titan in 1996-1998 using near-infrared (centered at 2.1 microns) speckle imaging at the 10-meter W.M. Keck Telescope. The observations have a spatial resolution of 0.06 arcseconds. We detect bright clouds on three days in October 1998, with a brightness about $0.5 \%$ of the brightness of Titan. Using a 16-stream radiative transfer model (DISORT) to model the central equatorial longitude of each image, we construct a suite of surface albedo models parameterized by the optical depth of Titan's hydrocarbon haze layer. From this we conclude that Titan's equatorial surface albedo has plausible values in the range of $0-0.20$. Titan's minimum haze optical depth cannot be constrained from this modeling, but an upper limit of 0.3 at this wavelength range is found. More accurate determination of Titan's surface albedo and haze optical depth, especially at higher latitudes, will require a model that fully considers the 3-dimensional nature of Titan's atmosphere.
\end{abstract}




\section{Introduction}

Unlike other known planetary satellites, Titan possesses a thick (1.5 bars) atmosphere. Observations by Voyager I in 1980 (Smith et al. 1981) at visible wavelengths showed that Titan was covered by a hydrocarbon haze with a pronounced north/south asymmetry, which hid the surface from view. However, Titan's surface can be imaged in the visible (at low contrast) and in the near-infrared at certain wavelengths where methane absorption windows occur. Thanks to new highresolution infrared imaging techniques, in the last decade we have learned some of the details of Titan's surface. Titan has been observed in visible and near-infrared windows by the HST (Meier et al. 2000, Smith et al. 1996), and in the infrared using adaptive optics technology (Combes et al. 1997, Coustenis et al. 2001). We have previously published the results of our observations of Titan in 1996 (Gibbard et al. 1999) using speckle imaging at the W.M. Keck Telescope.

The surface albedo of Titan is an important clue both to the present composition of its surface and to the history of Titan's surface-atmosphere interactions. Although theories (Lunine et al. 1983, Yung et al. 1984) predict that Titan's haze, produced by methane photolysis, should 'rain out' from the atmosphere to produce a thick surface layer of liquid hydrocarbons (mainly ethane), near-infrared and radar observations indicate that Titan does not possess a global ocean (e.g. Muhleman et al. 1991,1995; Griffith et al. 1991). This implies that either there are less hydrocarbons on the surface than theories predict (and possibly that Titan's atmosphere is not in a long-term steady-state; Lorenz et al. 1997a), or that the hydrocarbon is stored in subsurface reservoirs.

Titan's haze has a large optical depth at visible wavelengths which decreases 
in the near-infrared. Titan's haze optical depth varies as a function of latitude; in 1996-1998 at 2 microns Titan's atmosphere was brightest in the southern hemisphere. Extrapolations from observations suggest that Titan's haze infrared optical depth in the south should have reached a maximum some time around 1995-1996, with a subsequent decline predicted until 2010 (Lorenz et al. 1997b). Continuing observations of Titan at multiple wavelengths are needed to determine how Titan's haze optical depth changes in time.

The work presented here expands on our previous observations, including full longitude coverage of Titan at 2.1 microns for the period from 1996-1998. Using a radiative transfer model (DISORT) we estimate the contribution to our images from Titan's atmosphere, and thus place limits on Titan's plausible equatorial surface albedo and atmospheric haze optical depth. Our goals in this paper are: (1) to constrain Titan's equatorial surface albedo at 2 microns, (2) to determine limits on Titan's haze optical depth in this wavelength range, and (3) to report on evidence of the presence of clouds in Titan's southern hemisphere similar to those recently detected near Titan's south pole (Roe et al. 2002, Brown et al. 2002), but occurring several years earlier.

\section{Observations and Data Reduction}

Observations were made on September 6 1996, October 10-13 1997, July 27August 1 1998, and October 7-14 1998 (all dates UT) using the 10-m W.M. Keck I Telescope and the $256 \times 256$ pixel NIRC camera in speckle imaging mode (Matthews and Soifer 1994). The plate scale is 0.0203 arcseconds per pixel, which gives approximately 40 pixels across the disk of Titan. The data reported here were obtained in the $\mathrm{K}^{\prime}$ band $(1.95 \mu m-2.29 \mu m)$. This wavelength range includes both a strong 
methane absorption band and a window where methane absorption is nearly zero, so that light can penetrate to the surface. The spatial resolution we achieved was typically 0.06 arcseconds, which is $\simeq 375 \mathrm{~km}$ on Titan's surface. The observations are summarized in Table 1.

[Table 1 here]

Each data set consists of 100 frames, each with an exposure time of 150 or 200 milliseconds. Each night's data contains between 4 and 12 sets of 100 frames, so that the total number of frames used in each night's image is 400-1200. Each frame also has 100 dark/bias exposures associated with it, which are used to calibrate drifts in the DC bias level of NIRC's electronics. Our procedure for preprocessing the images, including sky and dark subtraction and destriping, was described in Gibbard et al. 1999.

After preprocessing, the data were processed using speckle imaging techniques. Speckle imaging is a high resolution technique that works well on bright objects such as Titan. The idea is to take very short exposures to "freeze" the atmospheric turbulence and capture the light while it is still forming coherent interference patterns at the detector. The coherence patterns contain diffraction-limited information that can subsequently be used to determine the object's Fourier amplitudes and phases, which are combined with an inverse Fourier transform to produce a diffractionlimited image. Algorithms for the recovery of diffraction-limited information were first introduced by Labeyrie (1970) for computing the Fourier amplitudes, or equivalently the autocorrelation of the image, and extended to full image recovery by Knox and Thompson (1974), Weigelt (1977), Lohmann et al. (1983), and Roddier (1986), who recognized the similarity of the bispectrum imaging method to phase closure techniques in radio interferometry. 
Table 1 gives information on the number of individual exposures combined to make up each image, the point spread function (PSF) star used, the photometric standard star used to calculate Titan's geometric albedo, the sub-Earth and subsolar latitude and longitudes in degrees, according to the 1997 IAU model (longitude of 0 is the sub-Saturn point, and longitude increases to the left in each image), the phase angle (Sun-Titan-Earth angle), the size of Titan's disk as seen from the Earth, and Titan's K' band geometric albedo for each date of observation. Figure 1 shows the images obtained from speckle reconstruction of the data for each of the 14 nights of observation.

[Figure 1 here]

The images have been rotated so that Titan north is up and increasing western Titan longitude is to the left. Thus the 'sunrise' limb of Titan appears to the left in each image.

In order to estimate the resolution of our data, we obtained speckle stacks of images of stars within a few hours of our Titan observations. These image stacks were then speckle processed in the same way as our Titan observations, using the same PSF star that was used for the Titan reconstruction (there were thus two stars observed for each night, one of which was used for the reconstruction and the other to determine the resolution). The star images obtained are shown in fig 2 .

[Figure 2 here]

We find that the typical full width at half maximum (FWHM) for these stars is about 0.06 arcseconds, ranging from $0.058-0.07$. This resolution is below the theoretical diffraction limit of 0.04 arcseconds at a wavelength of 2 microns, primarily because the speckle processing technique, like other deconvolution algorithms, in- 
troduces artifacts with over-application. We have chosen to minimize such artifacts at the cost of somewhat lower resolution than the theoretical diffraction limit.

\section{Detection of Clouds}

There is considerable evidence for methane supersaturation on Titan (Courtin et al. 1995, Samuelson et al. 1997), indicating that cloud formation is difficult. This may be due to a combination of low temperature and lack of suitable condensation nuclei. Tokano et al. (2001), who used a GCM to explore three-dimensional transport of methane in Titan's atmosphere, also found that methane supersaturation of at least $150 \%$ was most in agreement with Voyager data. Under conditions of supersaturation, their modeling predicts that condensation preferentially occurs at low latitudes at an altitude of $\simeq 15 \mathrm{~km}$.

The first observational evidence for tropospheric clouds on Titan came from variations in spectra of Titan's disk-integrated albedo (Griffith et al. 1998, 2000). Such clouds have now been directly detected by Roe et al. (2002) and Brown et al. (2002) using adaptive optics techniques with narrowband imaging on the Gemini and Keck Telescopes in December 2001. These clouds were located near Titan's south pole. The brightest clouds were also detected in a broadband K' filter, although this filter is not the best choice for cloud searches due to the lower contrast of the cloud features compared to their appearance in a narrower bandwith filter that selectively probes Titan's troposphere. Since Titan's southern hemisphere was near summer solstice at the time of these adaptive optics observations, the question arises whether this season was optimal for cloud formation due to an increase in surface temperature, and how far back in time such optimal cloud formation conditions might have existed. To address this question we examined our data for evidence of 
clouds.

Examination of our images from 14 nights of observation in 1996-1998 reveals one night which shows the presence of a cloud, and two nights which suggest the presence of clouds. Individual frames from the other nights were also examined but no consistent pattern (the same bright feature appearing in the majority of the speckle frames) was observed. Observations on October 14, 1998 show a bright feature on the left side of the disk (fig. 1) in the southern hemisphere. A similar feature is seen on October 9. On October 7 such a feature appears near the central meridian. We have considered three possibilities for the origin of these features: 1) they are artifacts of the speckle reduction process (which would be random, and would not appear at the same location in several frames), 2) they are surface features, and 3) they are cloud features.

In order to test the first possibility we have examined the frame stacks used to construct the images in fig. 1. The image from October 14 shown in fig. 1 was constructed from 8 stacks of 100 frames each, which were averaged to produce the final image. Figure 3 shows all 8 of the 100-frame stacks; the bright feature on the left-hand limb appears in all of them, and is indeed the brightest feature in the images.

[Figure 3 here]

We have also found that the bright feature seen on October 7 appears in all the 100 -frame stacks, but there were only 4 stacks taken on that night. The bright feature seen on October 9, though it appears in 8 of the 10 100-frame stacks, is not as clear a candidate as the October 14 feature since it does not appear in all the frames, and it is not of consistent brightness. 
Our rejection of these bright spots as surface features stems from the fact that observations on other nights do not show these bright surface features. Data obtained in 1999-2001 using adaptive optics (Gibbard et al. 2002) also do not show evidence for bright surface features at these locations.

We have calculated the fraction of the light from Titan in the K' filter that is due to the presence of the features on these dates; for October 7 the fraction is $0.6 \%, 0.4 \%$ for October 9 , and $0.5 \%$ for October 14 . These are estimates based on comparison with the opposite limb of Titan for October 9 and 14, and by comparison with limb profiles for other nights in the case of the October 7 data (where the feature occurs near the south pole). The daily clouds detected by Griffith et al. (2000) were calculated to occur over $\leq 1 \%$ of the satellite, while a cloud detected in 1995 was much brighter, covering 5-7\% of the disk (Griffith et al. 1998). Since the clouds appear to be resolved in our images, we can calculate a rough size estimate. The procedure we follow is to first project the images onto a latitude/longitude grid. We then convolve an artificial 'bright spot' of varying size with the observed PSF for the night in question, and subtract this artificial spot, scaled to the brightness of the bright feature, until the brightness is reduced to that of the opposite limb. The size of the spot needed to accomplish this is taken as the rough size of the bright feature. By this procedure we determined that the October 14 cloud has a total area of approximately 18 pixels, or about $1 \%$ of of the disk area. The October 9 cloud has a similar size, while the October 7 cloud is somewhat larger, covering about $1.5 \%$ of the disk.

\section{Atmosphere Model}

Since our data at K' band contain contributions from Titan's surface and at- 
mosphere, it is necessary to model the data using a radiative transfer code that simulates the absorption, reflection and scattering of photons from Titan's surface and atmosphere. We have in the past modeled Titan's atmosphere using a 1-D radiative transfer model (Gibbard et al. 1999). This type of model works well when the atmospheric curvature is small, so that the incoming or outgoing parallel beam of radiation has an angle of incidence with respect to the vertical direction of less than $80^{\circ}$. However, these conditions are not met for high spatial resolution images of Titan. Since the depth $(\sim 1000 \mathrm{~km})$ of Titan's atmosphere is comparable to its radius $(\sim 2575 \mathrm{~km})$, a 3 -D radiative transfer model for the solar flux transport within the atmosphere is necessary for a realistic simulation of the radiation flow in Titan's atmosphere near the limbs of the satellite (Lebonnois and Toublanc, 1999). However, it is still true that 1-D models are appropriate near the center of Titan's disk, where the incoming or outgoing beams make only small angles to the vertical. Since the central longitudes of our images range from $3-290^{\circ}$, we can, by modeling the center of each image, obtain information about the near-equatorial haze optical depth and surface albedo of Titan over most of the satellite's longitude range.

Our modeling was done as follows: for each image the I/F (ratio of the observed intensity $\mathrm{I}$ to $1 / \pi$ of the incoming solar radiation $\pi \mathrm{F}$ ) at the central meridian was calculated. A 3 X 3 pixel region of each image was averaged to correspond to our spatial resolution of 0.06 arcseconds. The average central I/F at each date of observation is shown in Table 2. Since the sub-Earth latitude varied, the equator was not always located at the center of the images; however in every case it was within 16.6 degrees of the center (the maximum sub-Earth latitude being $-16.6^{\circ}$ ), in which range the 1-D radiative transfer approximation should still be valid. For the case of a central longitude of $125^{\circ}$ we have two measurements made in 1996 and 
1997; these were averaged together to obtain the modeled value.

[Table 2 here]

To calculate photon transport we used two radiative tranfer codes: DISORT (Stamnes et al. 1988), which is a multi-stream model, and the modified two-stream model described by Toon et al. 1989, which we used in our previous modeling of Titan (Gibbard et al. 1999). This model has also been applied to Titan by Toon et al. (1992), and Hutzell et al. (1993, 1996). Our nominal DISORT model used 16 streams; the effect of using a smaller number of streams is discussed below.

Our model atmosphere is divided into 2 vertical layers, each of which has a value for the haze and gas optical depths. The haze is confined to the top layer, with methane underneath. The methane is assumed to be completely absorbing within the fraction of the filter that is in the methane band (this fraction is estimated to be $59 \%$ for K', based on measurements taken at $206 \mathrm{~K}$ at $0.5 \mathrm{~km}$ amagat (Figure 4 ; Caitlin Griffith, personal communication)), and to be

[Figure 4 here]

transparent outside of the methane absorption band. (The effect of a higher amount of atmospheric absorption was also considered, as discussed below). Scattering parameters for the haze are as described in Gibbard et al. 1999: a scattering asymmetry factor of 0.2 and a single scattering albedo of 0.85 . The single criterion for goodness of fit is that the model should reproduce the equatorial I/F seen in our images of Titan for a given longitude. For each image there are two free parameters: the surface albedo and the haze optical depth. Obviously then we cannot uniquely determine either variable. However, under the assumption that Titan's equatorial haze optical depth remained roughly constant over the period when our 
images were taken, the surface albedo at different longitudes around the equator can be estimated provided that a single values for the haze optical depth is adopted. If the optical depth does vary significantly as a function of time, the situation is more complicated, because the surface albedo at each latitude can only be specified provided the appropriate haze optical depth for each date of observation is known.

\section{Equatorial Surface Albedo}

Titan's surface albedo provides clues to its surface composition. In the past suggested surface compositions ranged from liquid hydrocarbon oceans (Lunine 1983) to solid rock or ice/hydrocarbon mixtures (Muhleman et al. 1991, Griffith et al. 1991). More recently Coustenis et al. (2001) suggested that the bright material on Titan probably contains more than one component, perhaps including methane or ethane frost and water ice as well as organics. Despite the likely presence of hydrocarbons on Titan's surface, there is evidence that ice is extensively exposed there (Griffith et al. 2003).

The most striking feature on Titan's surface is the bright feature on the leading hemisphere near $90^{\circ}$ longitude. This feature was noted by Lemmon et al. (1993) and Griffith (1993) as a variation in Titan's near-infrared disk-averaged albedo, and was resolved by the HST (Smith et al. 1996), speckle imaging (Gibbard et al. 1999) and adaptive optics images (Coustenis et al. 2001, Combes et al. 1997). The equatorial region of the trailing hemisphere appears to be considerably darker.

[Figure 5 here]

Figure 5 shows the equatorial surface albedo derived for the central meridian longitude of each image as a function of the haze optical depth. Note that we do not 
have images centered directly on $90^{\circ}$ longitude, the center of Titan's bright feature, so the true peak albedo may be somewhat higher than the albedo found for $125^{\circ}$. The possible surface albedo ranges from near 0 for the darkest regions of the trailing hemisphere for the case of $\tau=0.3$ to a maximum of $\simeq 0.2$ for a longitude of $125^{\circ}$ under the (unrealistic) assumption of $\tau=0$. If we take the value of $\tau$ to be close to that given by Griffith et al. (2003) (about 0.2) then the surface albedo at the given central longitudes ranges from 0.03 to 0.16 . However, Titan's haze optical depth is not uniform in latitude, and the value of 0.2 may not accurately characterize the haze optical depth at the equator. Also, the haze optical depth is varying in time as the seasons change on Titan (Lorenz et al. 2001).

\section{Titan's haze optical depth at 2 microns}

When Voyager I visited Titan in 1980, the northern hemisphere was darker than the south at visible wavelengths (Sromovsky et al. 1981) which implies greater haze optical depth in the north at this time; in the 1990's, when the spatial resolving power of the Hubble Space Telescope became available, it was found that the brightness asymmetry had reversed (Caldwell et al. 1992, Lorenz et al. 1997b). It is not known, however, whether this reversal takes place gradually, or occurs as a relatively rapid change. Also not known is the exact mechanism that produces the change. It appears that the albedo difference is not driven by an increase in the haze production rate, since the response of the haze to changes in production rate is too slow (Hutzell et al. 1993). The data seem to be fit best by a change in number density of the haze with altitude, driven by circulation (Tokano et al. 1999). Titan's haze distribution appears to consist of a general tropospheric haze, overlain by a detached higher-level haze in the winter hemisphere, and polar hoods (Rannou 
et al. 2002).

Although our current modeling does not allow us to calculate an exact value for Titan's haze optical depth, a maximum value can be determined by noting that for a value of $\tau=0.3$ the implied surface albedo at the darker longitudes of the planet is zero. This remains true even for the case where the fraction of the light from the surface in the $\mathrm{K}^{\prime}$ filter is as little as $20 \%$ (half the nominal value), as discussed below. Therefore we believe that, given the assumptions we have made, the equatorial haze optical depth on Titan during this time period did not exceed 0.3. A minimum value for $\tau$ is not obvious, although it does have a value significantly greater than zero, evident from Titan's strong limb brightening. A model using spherical particles (McKay et al. 1989) predicted a value of 0.5 for the haze optical depth at 2.1 microns, compared to a predicted value of 0.3 for fractal particle models (Rannou et al. 1995). It thus appears that fractal models are more consistent with the observed haze optical depth on Titan.

\section{Discussion}

Errors. There are several possible sources of error in our calculations. First, there is error due to inaccuracies in the radiative transfer modeling. Second are errors resulting from the use of parameterized haze models, with parameters whose accuracy is uncertain. Lastly, there are night-to-night variations due to variations in observing conditions and the resulting accuracy of photometry and deconvolution of the speckle images. The errors in our photometry (based on multiple observations of a reference star) varied from night to night but were typically on the order of $10 \%$. 
Our nominal model used a 16-stream version of the DISORT code (Stamnes et al. 1988). We also performed calculations using 4-stream and 8-stream DISORT models, as well as a modified 2-stream model (Toon et al. 1989) that we have used in previous modeling of Titan's atmosphere (Gibbard et al. 1999). Results from the comparison of the 4-stream model to the nominal model showed that differences in the central disk I/F ranged from 0.6 to $5.3 \%$ (the I/F for the 4 -stream model being lower in every case), while the 8-stream model had an I/F that was lower than the 16 -stream model by 0.01 to $0.6 \%$. Therefore in this case an 8 -stream model does appear to be comparably accurate to the 16-stream model. Results for the Toon et al. model were more mixed, as shown in the comparison in Table 3. The Toon et al. model typically give higher central disk albedoes for low values of the haze optical depth, and slightly higher values than the 16-stream DISORT models for larger values of $\tau$. In general agreement with the DISORT code improved for larger values of $\tau$.

\section{[Table 3 here]}

Another parameter we have adopted that is significantly uncertain is the fraction of light in the K' band that actually comes from Titan's surface. In our nominal model this fraction is $41 \%$, based on integration of a methane absorption spectrum taken at $206 \mathrm{~K}$ at $0.5 \mathrm{~km}$ amagat (fig. 4). However, since there is uncertainty about the methane spectrum for temperatures and pressures that correspond to Titan's atmosphere, the actual fraction of light from the surface may differ. To get a feel for the difference this would make in our derived surface albedoes, we have also considered models in which the fraction of light from the surface is only $20 \%$. Results from this modeling are shown in Fig. 6. In this case surface albedoes would obviously be higher, ranging up to even 0.3 or 0.4 in the brightest regions. However, 
note that for $\tau=0.3$ the surface albedo of the darkest regoins would still be zero. For a haze optical depth of 0.2 the surface albedo would range from 0.07 to 0.32 .

[Figure 6 here]

Clouds. The first claim of cloud detection on Titan was based on spectroscopic evidence from Griffith et al. (1998), work which later indicated the detection of daily clouds (Griffith et al. 2000). Clouds have recently been detected in Gemini and Keck adaptive optics observations by Roe et al. (2002) and Brown et al. (2002). These clouds occur in Titan's southern hemisphere, and the deduced altitude (Griffith et al. 1998, Brown et al. 2002) is $16 \mathrm{~km}$, in the middle of Titan's troposphere (the fainter clouds detected by Griffith et al. 2000 were calculated to occur at a higher altitudes of $\simeq 27 \mathrm{~km}$ ). This is similar to the altitude suggested by Tokano et al. (2001) for maximum likelihood of cloud formation in a supersaturated atmosphere. It was suggested by Brown et al. 2002 that the higher temperature at Titan's south pole during summer solstice could cause the atmosphere to be marginally unstable to convection, triggering the formation of clouds. Alternatively, or additionally, Roe et al. (2002) postulates the presence of ethane-coated particles in the troposphere near the south pole which rain out from the polar stratospheric haze cap. Such particles could act as condensation nuclei for methane gas, driving moist convection. On the other hand, the clouds considered by Tokano et al. (2001) would form in a highly supersaturated region, and would have a very short lifetime. In this case, the clouds would evaporate before reaching the surface, and Titan's hydrological cycle would take place entirely within its atmosphere.

Our detection of possible clouds on Oct. 7,9, and 14 1998, and the absence of such features from our observations in 1996, 1997, and July 1998, indicates that clouds (or at least distinct bright cloud features) may have been episodic, occuring 
over timescales of days or weeks. The detection of such clouds indicates that favorable conditions existed for cloud formation at the south pole at least several years before the summer solstice in October 2002. If the Griffith et al. (1998) bright cloud seen in 1995 also occurred in the southern hemisphere, this would indicate that clouds may occur long before the time of maximum insolation, and are not caused solely by a very high temperature at the south pole. It suggests that the presence of condensation nuclei is more important, in which case the dissipation of the southern polar haze cap could lead to the end of cloud formation there. This theory also predicts that the formation of haze over the northern pole would lead to the formation of clouds in the northern hemisphere after the particles have had enough time to fall down to the troposphere.

Titan surface albedo. Based on single-wavelength observations it is impossible to determine the surface composition of Titan. Observations by Coustenis et al. (1995) indicated that Titan's surface albedo was compatible with the presence of water ice and another component, probably organics. Their later observations at several different infrared wavelenths (Coustenis et al. 2001) suggest that the bright material on Titan's leading hemisphere is not simply water ice, which should not appear bright at both 1.6 and 2 microns. Coustenis et al. speculate that that the bright material may consist of methane or ethane frost and water ice, probably combined with organic material. Recent observations by Griffith et al. (2003) indicate that Titan's disk-integrated albedo over a large range of wavelengths is consistent with exposed water ice.

Titan's surface albedo at 2 microns has been estimated by previous researchers, including Coustenis et al. 1995 (0.24 for the bright terrain and 0.17 for the dark terrain), Lemmon et al. 1995 (0.27 for the bright terrain and 0.07 for the dark 
terrain), Griffith et al. 2003 (0.12 for the leading hemisphere and 0.08 for the trailing hemisphere), and Bouchez et al. 2000 (values ranging from 0.07-0.15). Based on our modeling the most likely surface albedo values for our nominal model are in the range of $0.15-0.2$ for the brightest and $0.04-0.08$ for the darkest equatorial regions.

Although there is currently some variation in the albedo values found by different observers, one thing that is clear from all observations is that Titan's surface is heterogenous and that there is a large albedo contrast between features such as the bright region near $90^{\circ} \mathrm{W}$ longitude and the much darker equatorial region on the trailing hemisphere.

Haze optical depth. Based on the increased limb brightening in the south compared to the north, easily visible in fig. 1, our observations from 1996-1998 indicate greater haze optical depth at $\mathrm{K}$ band in the southern hemisphere than in the north. The north/south haze asymmetry is believed to be a seasonal effect, with maximum haze optical depth shifting from pole to pole during Saturn's 30-year orbital period around the Sun (Lockwood et al. 1986). Voyager observations showed that the northern hemisphere of Titan was darker than the south at visible wavelengths in 1980 (Sromovsky et al. 1981). This implies greater haze optical depth in the north at that time for visible wavelengths, which probe upper layers in Titan's atmosphere. Observations by the HST over the period from 1994-2000 (Lorenz et al. 2001) indicate that seasonal changes in Titan's haze distribution have occurred at visible and near-infrared wavelengths up to $889 \mathrm{~nm}$. However, Lorenz et al. find that filters which probe more deeply into the atmosphere, such as 600-700 nm and 820 and $940 \mathrm{~nm}$, had not shown a reversal in haze asymmetry. They suggest that Titan's seasonal cycle of haze redistribution has an altitude-dependent phase lag, 
such that the haze at higher altitudes is redistributed before haze at lower altitudes.

Our own observations support the idea that Titan's haze asymmetry had not yet (as of 1998) shown a reversal at $\mathrm{K}$ band (2 microns), a filter that probes relatively deeply into the atmosphere. The constraint we derive for the equatorial haze optical depth $(\leq 0.3)$ is consistent with values given by Griffith et al. $(1991,2003)$ and by Bouchez et al. (2000) (0.04 in the northern mid-latitudes and 0.2 above the south pole). Both our values and those of Bouchez et al. and Griffith et al. are lower than predicted from either spherical or fractal haze particle models (McKay et al. 1989 and Rannou et al. 1995).

\section{Conclusions}

Observations of Titan using speckle imaging at the W.M. Keck I Telescope allow us to obtain excellent spatial resolution (0.06" across the disk of Titan) at a wavelength range that probes both Titan's surface and atmosphere. These images show a variety of surface features on Titan, and yield constraints on the haze optical depth in Titan's atmosphere. They also suggest the presence of episodic clouds in the southern hemisphere, in agreement with recent adaptive optics observations (Brown et al. 2002, Roe et al. 2002). Titan's north/south asymmetry in atmospheric brightness has continued to be pronounced at 2 microns in the period from 1996-1998. Since HST observations indicate that this asymmetry had begun to reverse at shorter wavelengths during this time, this suggests a difference in haze density in the two hemispheres at a relatively low altitude. Variations in surface reflectance, on scales that can only be resolved by high-resolution imaging, indicate a highly heterogenous surface. This includes equatorial bright areas with an albedo that we found to be $0.15-0.20$, and darker areas with reflectance $<0.08$ which 
might be covered by deposits of organic solids or liquid hydrocarbons. More accurate modeling of Titan's atmosphere and surface awaits an accurate determination of Titan's atmospheric structure (which should be provided by the Cassini Huygens probe in early 2005), as well as a radiative transfer model that fully considers the 3-dimensional nature of Titan's atmosphere. 


\section{ACKNOWLEDGEMENTS}

Our thanks to referees Athena Coustenis and Peter Smith who provided many useful suggestions for the improvement of this manuscript.

Data presented herein were obtained at the W.M. Keck Observatory, which is operated as a scientific partnership among the California Institute of Technology, the University of California and the National Aeronautics and Space Administration. The Observatory was made possible by the generous financial support of the W.M. Keck Foundation. The authors wish to recognize and acknowledge the very significant cultural role and reverence that the summit of Mauna Kea has always had within the indigenous Hawaiian community. We are most fortunate to have the opportunity to conduct observations from this mountain.

This research was partially supported by the NASA Planetary Astronomy Program under grant number NRA-97-OSS-04-98-053(R3281), and by the National Science Foundation grant AST-0205893 to the University of California, Berkeley.

This work was supported in part by the National Science Foundation Science and Technology Center for Adaptive Optics, managed by the University of California at Santa Cruz under cooperative agreement No. AST-9876783.

This work was performed under the auspices of the U.S. Department of Energy, National Nuclear Security Administration by the University of California, Lawrence Livermore National Laboratory under contract No. W-7405-Eng-48. 


\section{FIGURE CAPTIONS}

Fig. 1: Reconstructed speckle images from each of the 14 nights of observation. Sky north is up, sky east (the direction of increasing Titan longitude) is to the left. Details on each image can be found in Table 1.

Fig. 2: Images of stars observed within a few hours of each Titan observation, indicating the stars' full width half maximum, in arcseconds. The stars were speckleprocessed using the same procedure used for the images.

Fig. 3: The full set of 100-frame stacks obtained on October 14, 1998, showing the presence of a bright feature near the southern left limb of the satellite. These stacks were added together to produce the final image shown in Fig. 1.

Fig. 4: Methane transmission as a function of wavelength over the $\mathrm{K}$ ' band. An integration of this spectrum leads to the nominal number of $41 \%$ transmission over the K' band. Figure is based on a methane absorption spectrum taken at $206 \mathrm{~K}$ at $0.5 \mathrm{~km}$ amagat (Caitlin Griffith, personal communication).

Fig. 5: Equatorial surface albedo as a function of latitude on Titan. The albedoes are derived from the central latitude of each image, as described in the text. Assuming a constant equatorial haze optical depth, albedo curves can be constructed for each value of $\tau$. Note that a maximum $\tau$ of 0.3 is implied by the fact that larger $\tau$ would give a negative surface albedo.

Fig. 6: Equatorial surface albedo as a function of latitude on Titan, assuming a model where the surface contribution to the images is $20 \%$ (compared to the nominal value of $41 \%$ ). This leads to higher maximum surface albedoes (compare to fig. 5), and more variation between the minimum and maximum albedoes for a 
given value of $\tau$. As in fig. 5, a maximum $\tau$ of 0.3 is implied. 


\section{REFERENCES}

Bouchez, A.H., M.E. Brown, C.A. Griffith, and R.G. Dekany. 2000. Spatially resolved spectroscopy of Titan's surface and atmosphere. American Astronomical Society, DPS Meeting \#32, \#17.02.

Brown, M.E., A.H. Bouchez, and C.A. Griffith. 2002. Direct detection of variable tropospheric clouds near Titan's south pole. Nature 420, 795-797.

Caldwell, J.D., P.H. Smith, M.G. Tomasko, and H. Weaver. 1992. Titan: evidence of seasonal change-A comparison of Voyager and Hubble Space Telescope images. Icarus 103, 1-9.

Combes, M. L. Vapillon, E. Gendron, A. Coustenis, O. Lai, R. Wittemberg, and R. Sirdey. 1997. Spatially resolved images of Titan by means of adaptive optics. Icarus $\mathbf{1 2 9}$, 482-497.

Courtin, R., D. Gautier, and C.P. McKay. 1995. Icarus 114, 144-162.

Coustenis, A., E. Gendron, O. Lai, J.-P. Veran, J. Woillez, M. Combes, L. Vapillon, T. Fusco, L. Mugnier and P. Rannou. 2001. Images of Titan at 1.3 and $1.6 \mu \mathrm{m}$ with Adaptive Optics at the CFHT. Icarus 154, 501-515.

Coustenis, A., E. Lellouch, J.P. Maillard, and C.P. McKay. 1995. Titan's surface: composition and variability from the near infrared albedo. Icarus 118, 87-104.

Gibbard, S.G., B.A. Macintosh, C.E. Max, I. de Pater, F. Marchis, H.G. Roe, D.S. Acton, O. Lai, P.L. Wizinowich, P. Stomski, E.F. Young, and C.P. McKay. 2002. Observations of Titan at $2 \mu \mathrm{m}$ with the W.M. Keck Telescope adaptive optics system. Submitted to Icarus.

Gibbard, S.G., B. Macintosh, D. Gavel, C.E. Max, I. de Pater, A.M. Ghez, E.F 
Young, and C.P. McKay. 1999. Titan: high-resolution speckle images from the Keck Telescope. Icarus 139, 189-201.

Griffith, C.A., T. Owen, T.R. Geballe, J. Rayner, and P. Rannou. 2003. Evidence for the exposure of water ice on Titan's surface. Science 300, 628-630.

Griffith, C.A., J.L. Hall, and T.R. Geballe. 2000. Detection of daily clouds on Titan. Science 290, 509-513.

Griffith, C.A., T. Owen, G. A. Miller, and T. Geballe. 1998. Transient clouds in Titan's lower atmosphere. Nature 395, 575-578.

Griffith, C.A. 1993. Evidence for surface heterogeneity on Titan. Nature 364, $511-514$.

Griffith, C.A., T. Owen, and R. Wagener. 1991. Titan's surface and troposphere, investigated with ground-based near-infrared observations. Icarus 93, 362-378.

Hutzell, W.T., C.P. McKay, and O.B. Toon. 1993. Effects of time-varying haze production on Titan's geometric albedo. Icarus 105, 162-174.

Hutzell, W.T., C.P. McKay, O.B. Toon, and F. Hourdin. 1996. Simulations of Titan's brightness by a two-dimensional haze model. Icarus 119, 112-129.

Knox, K. T. and B.J. Thompson. 1974. Recovery of images from atmospherically degraded short-exposure photographs. Astrophys. J. 193, L45-48.

Labeyrie, A. 1970. Attainment of diffraction limited resolution in large telescopes by Fourier analysing speckle patterns in star images. Astron. Astophys. 6, 85-87.

Lebonnois, S., and D. Toublanc. 1999. Actinic fluxes in Titan's atmosphere, from one to three dimensions: Application to high-latitude composition. JGR 104, 22025-22034. 
Lemmon, M.T., E. Karkoschka, and M. Tomasko. 1995. Titan's rotational lightcurve.I Icarus 113, 27-38.

Lemmon, M.T., E. Karkoschka, and M. Tomasko. 1993. Titan's rotation: surface feature observed. Icarus 103, 329-332.

Lockwood, G.W., B.L. Lutz, D.T. Thompson, and S.E. Bus. 1986. The albedo of Titan. Astrophys. J. 303, 511-520.

Lohmann, A., G. Weigelt, and B. Wirnitzer. 1983. Speckle masking in astronomy - Triple correlation theory and applications. Applied Optics 22, 4028-4037.

Lorenz, R.D., E.F. Young, and M.T. Lemmon. 2001. Titan's smile and collar: HST observations of seasonal change 1994-2000. GRL 28, 4453-4456.

Lorenz, R.D., C.P. McKay, and J.I. Lunine. 1997a. Photochemically driven collapse of Titan's atmosphere. Science $\mathbf{2 7 5}, 642-644$.

Lorenz, R.D., P.H. Smith, M.T. Lemmon, E. Karkoschka, G.W. Lockwood, and J. Caldwell. 1997b. Titan's north-south asymmetry from HST and Voyager imaging: comparison with models and ground-based photometry. Icarus 127, 173-189.

Lunine, J.I., D.J. Stevenson, and Y.L. Yung. 1983. Ethane ocean on Titan. Science 222, 1229-1230.

Matthews, K., and B.T. Soifer. 1994. Infrared Astronomy with Arrays: the Next Generation. I. McLean, ed. (Dordrecht: Kluwer Academic Publishers), p. 239.

McKay, C.P., J.B. Pollack, and R. Courtin. 1989. The thermal structure of Titan's atmosphere. Icarus 80, 23-53.

Meier, R., B.A. Smith, T.C. Owen, and R.J. Terrile. 2000. The surface of Titan 
from NICMOS observations with the Hubble Space Telescope. Icarus 145, 462473.

Muhleman, D.O., A.W. Grossman, and B.J. Butler. 1995. Radar investigations of Mars, Mercury, and Titan. Annual Review of Earth and Planetary Science 23, 337-374.

Muhleman, D.O., A.W. Grossman, B.J. Butler, and M.A. Slade. 1991. Radar reflectivity of Titan. Science 248, 975-980.

Rannou, P., M. Cabane, E. Chassefiere, R. Botet, C.P. McKay, and R. Courtin. 1995. Titan's geometric albedo: role of the fractal structure of the aerosols. Icarus 118, 355-372.

Rannou, P., F. Hourdin, and C.P. McKay. 2002. A wind origin for Titan's haze structure. Nature 418, 853-856.

Roddier, F. 1986. Triple correlation as a phase closure technique. Optics Communications 60, 145-148.

Roe, H.G., I. de Pater, B.A. Macintosh and C.P. McKay. 2002. Titan's clouds from Gemini and Keck adaptive optics imaging. Ap. J. 581, 1399-1406.

Roe. H.G. 2002. 'Titan's Atmosphere at High-Resolution', PhD. Thesis, UC Berkeley.

Samuelson, R.E., N.R. Nath, and A. Borysow. 1997. Gaseous abundances and methane supersaturation in Titan's troposphere. Planet. Space. Sci 45, 959980.

Smith, P.H., M.T. Lemmon, R.D. Lorenz, L.A. Sormovksy, J.J. Caldwell and M.D. Allison. 1996. Titan's surface, revealed by HST imaging. Icarus 119, 336-349. 
Smith, B.A., L.A Soderblom, R. Beebe, J. Boyce, G. Briggs, A. Bunker, S.A. Collins, C.J. Hansen, T.V. Johnson, J.L. Mitchell, R.J. Terrile, M. Carr, A.F. Cook II, J. Cuzzi, J.B. Pollack, G.E. Danielson, A. Ingersoll, M.E. Davies, G.E. Hunt, H. Masursky, E. Shoemaker, D. Morrison, T. Owen, C. Sagan, J. Veverka, R. Strom, and V. Suomi. 1981. Encounter with Saturn: Voyager 1 imaging results. Science 212, 163-182.

Sromovsky, L.A., V.E. Suomi, J.B. Pollack, R.J. Kraus, S.S. Limaye, T. Owen, H. E. Revercomb, and C. Sagan. 1981. Implications of Titan's north-south brightness asymmetry. Nature 292, 698-702.

Stamnes, K., S.-C. Tsay, W. Wiscombe and K. Jayaweera. 1988. A Numerically Stable Algorithm for Discrete-Ordinate-Method Radiative Transfer in Multiple Scattering and Emitting Layered Media. Appl. Opt. 27, 2502-2509.

Tokano, T., F.M. Neubauer, M. Laube, and C.P. McKay. 2001. Three-dimensional modeling of the tropospheric methane cycle on Titan. Icarus 153, 130-147.

Tokano, T., F.M. Neubauer, M. Laube, and C.P. McKay. 1999. Seasonal variation of Titan's atmopsheric structure simulated by a general circulation model. Icarus 47, 493-520.

Toon, O.B., C.P. McKay, T.P. Ackerman, and K. Santhanam. 1989. Rapid calculation of radiative heating rates and photodissociation rates in inhomogenous multiple scattering atmospheres. J.G.R. 94, 16287-16301.

Toon, O.B., C.P. McKay, C.A. Griffith, and R.P. Turco. 1992. A physical model of Titan's aerosols. Icarus 95, 24-53.

Weigelt, G.P. 1977. Modified astronomical speckle interferometry 'speckle masking'. Optics Communications 21, 55-59. 
Yung, Y.L., M. Allen, and J.P. Pinto. 1984. Photochemistry of the atmosphere of Titan: comparison between model and observations. Astrophys. J. (Suppl. Ser.) $\mathbf{5 5}, 465-506$. 


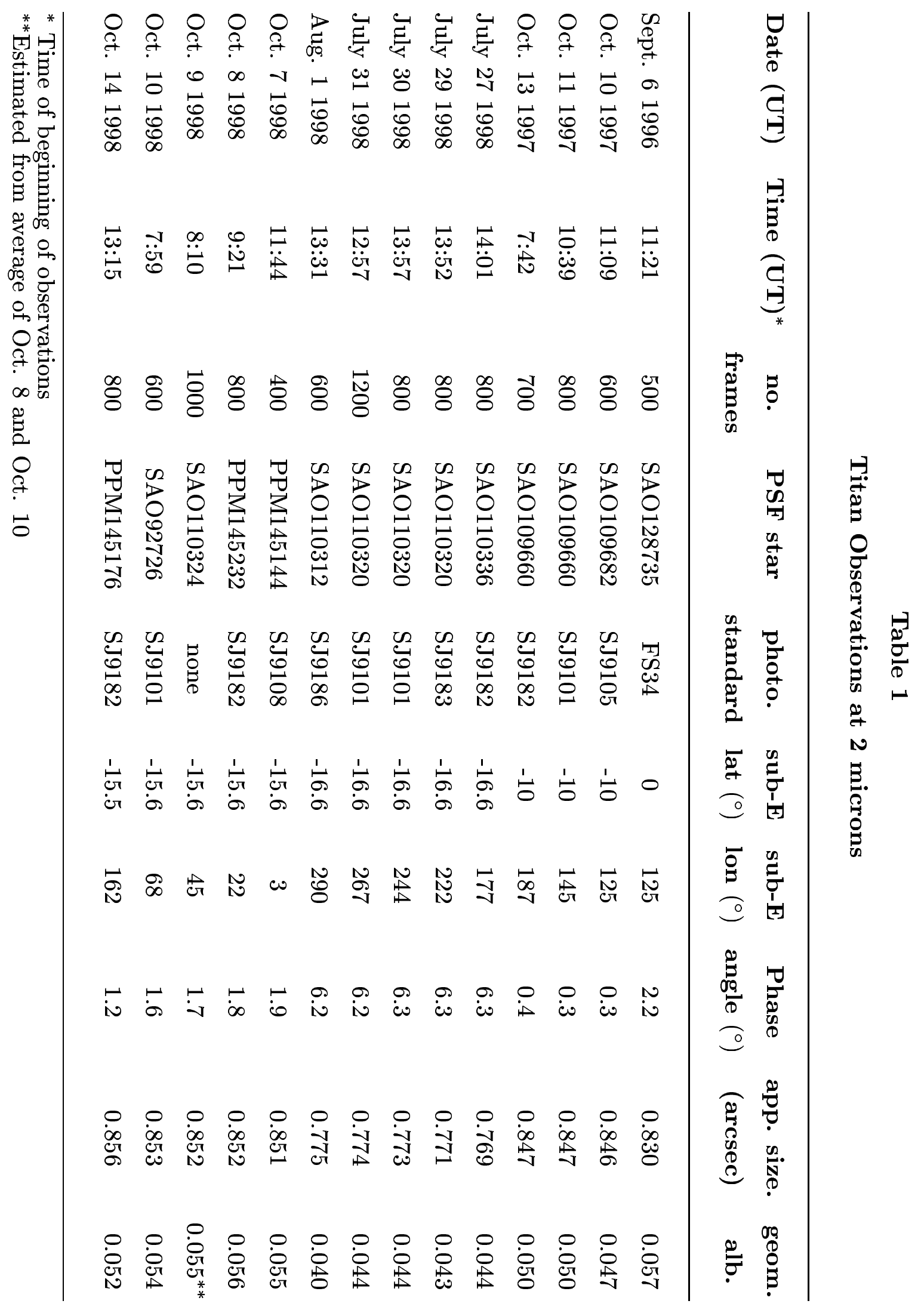


Table 2

Central I/F vs. Longitude

\begin{tabular}{lcc}
\hline Date & Central Longitude & Central Albedo \\
\hline Oct. 71998 & 3 & 0.057 \\
Oct. 81998 & 22 & 0.055 \\
Oct. 91998 & 45 & 0.063 \\
Oct. 101998 & 68 & 0.058 \\
Sept. 61996 & 125 & 0.084 \\
Oct. 101997 & 125 & 0.078 \\
Oct. 111997 & 145 & 0.051 \\
Oct. 141998 & 162 & 0.056 \\
Jul. 27 1998 & 177 & 0.050 \\
Oct. 131997 & 187 & 0.036 \\
Jul. 29 1998 & 222 & 0.057 \\
Jul. 301998 & 244 & 0.038 \\
Jul. 311998 & 267 & 0.048 \\
Aug. 11998 & 290 & 0.045 \\
\hline
\end{tabular}


Table 3: Comparison of Titan Central Albedo for 2 Radiative Transfer Models

\begin{tabular}{cccccc}
\hline & S. alb. $=\mathbf{0}$ & $\mathbf{0 . 0 5}$ & $\mathbf{0 . 1}$ & $\mathbf{0 . 1 5}$ & $\mathbf{0 . 2}$ \\
\hline $\begin{array}{c}\tau=0.05, \text { Toon et al. model } \\
\text { DISORT model }\end{array}$ & 0.02 & 0.04 & 0.05 & 0.07 & 0.09 \\
& 0.01 & 0.02 & 0.04 & 0.06 & 0.08 \\
$\tau=0.1$, Toon et al. model & 0.02 & 0.04 & 0.06 & 0.07 & 0.09 \\
$\quad$ DISORT model & 0.01 & 0.03 & 0.05 & 0.07 & 0.08 \\
& & & & & \\
$\tau=0.2$, Toon et al. model & 0.03 & 0.05 & 0.06 & 0.08 & 0.09 \\
$\quad$ DISORT model & 0.02 & 0.04 & 0.06 & 0.07 & 0.09 \\
$\tau=0.3$, Toon et al. model & 0.04 & 0.05 & 0.06 & 0.08 & 0.09 \\
$\quad$ DISORT model & 0.04 & 0.05 & 0.07 & 0.08 & 0.10 \\
\hline
\end{tabular}



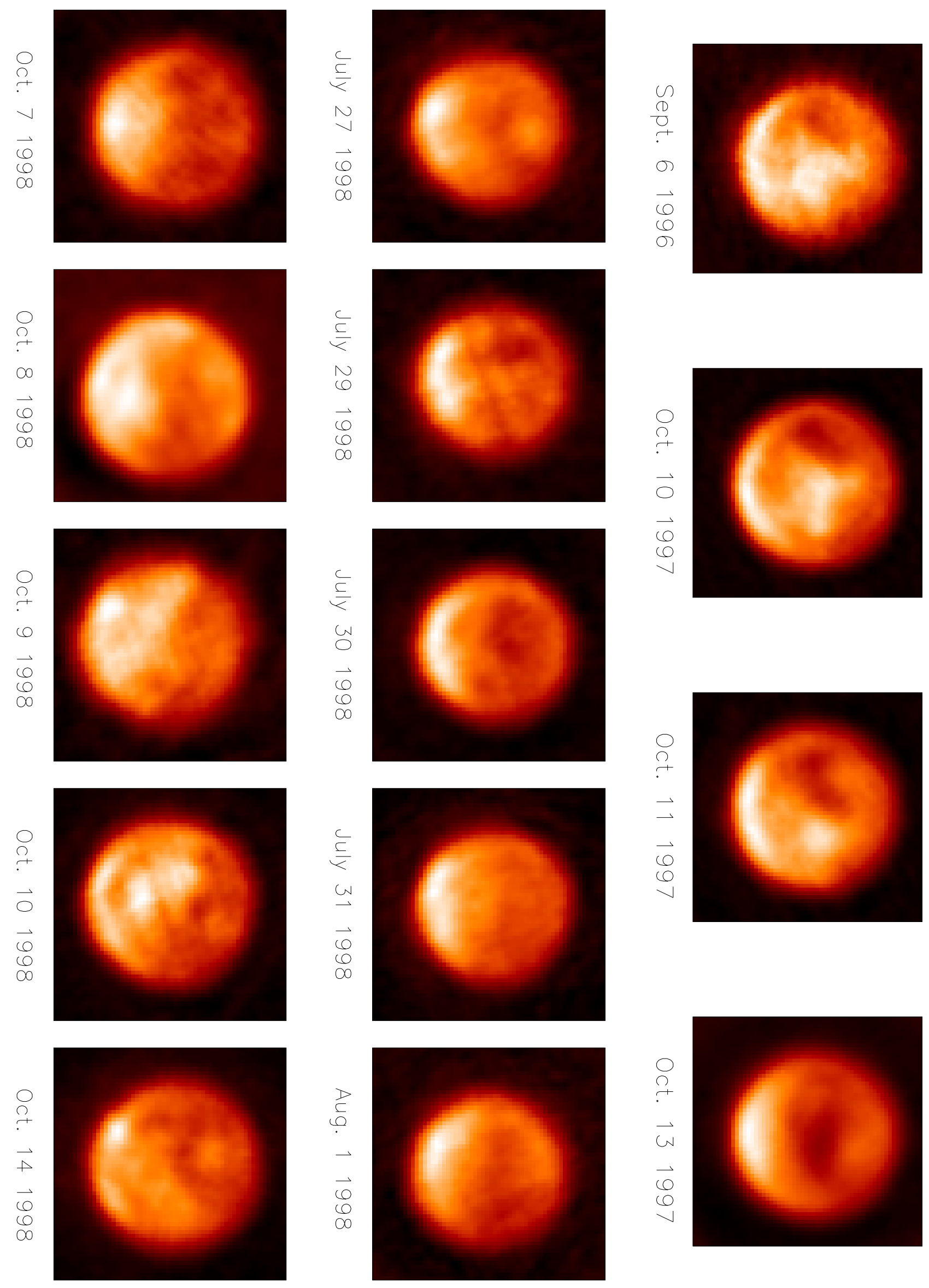

$\frac{\frac{T}{\sigma}}{\frac{5}{\pi}}$
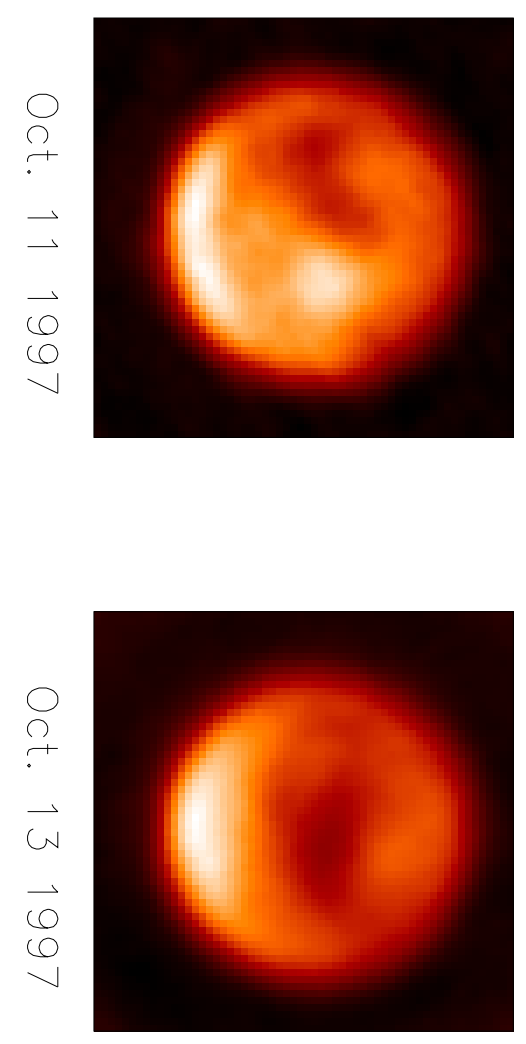

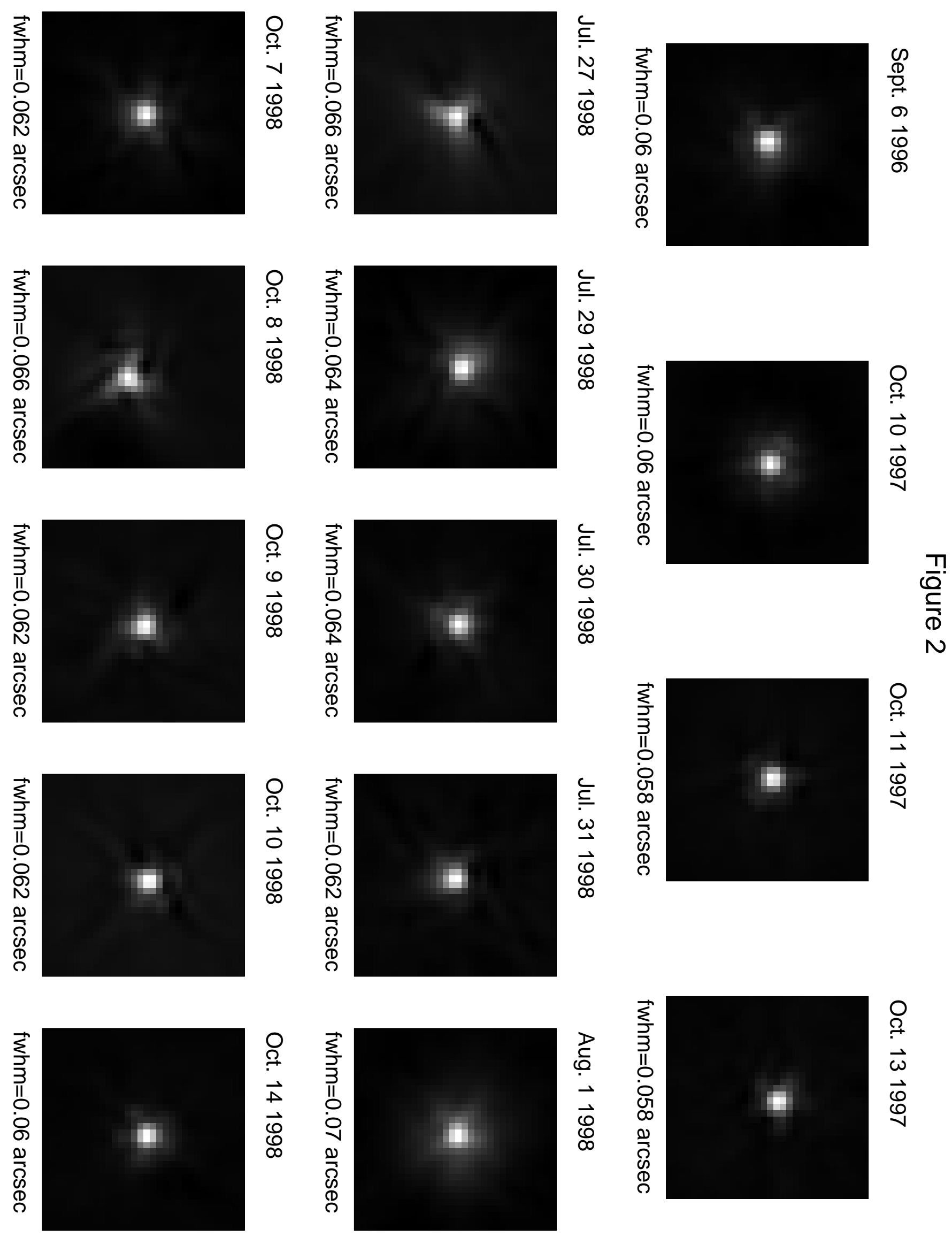

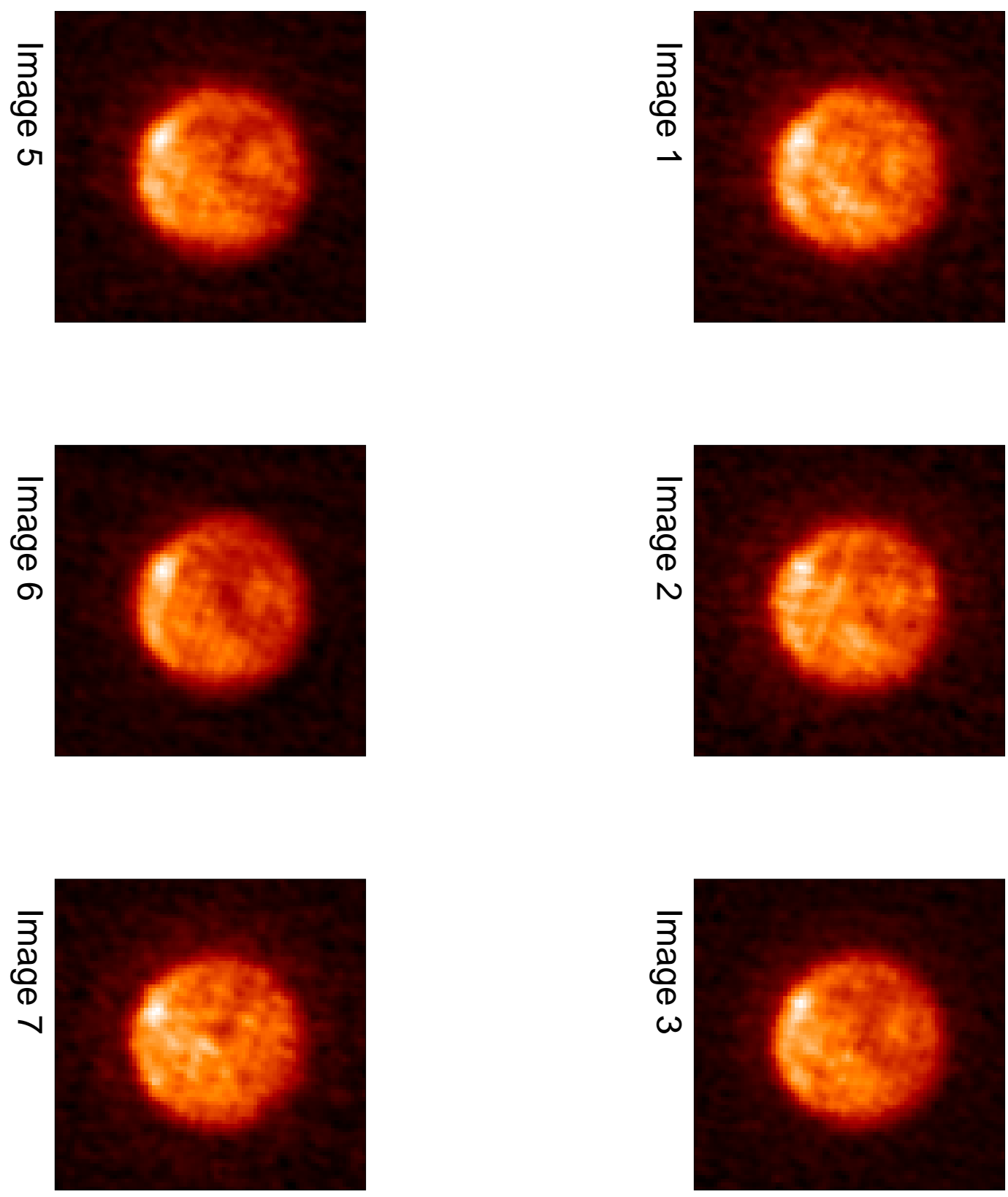

$\frac{T}{Q}$
$\frac{0}{\bar{D}}$
$\omega$
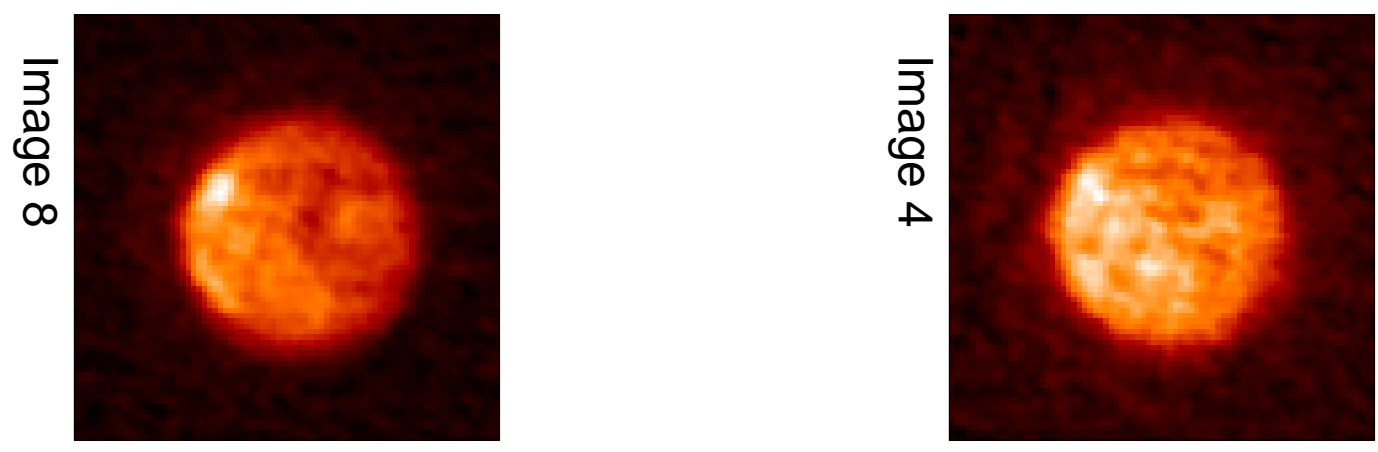


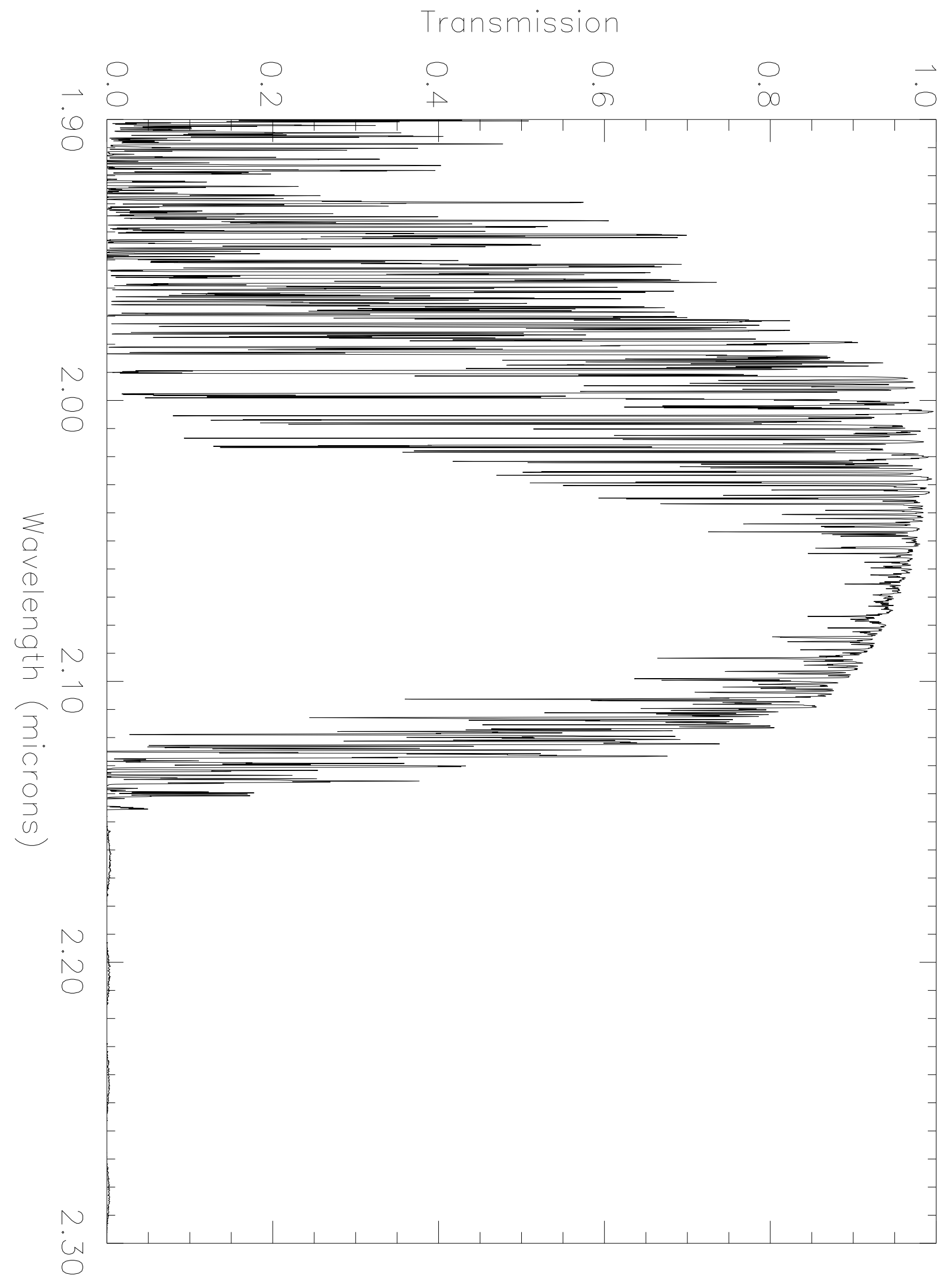


Figure 5

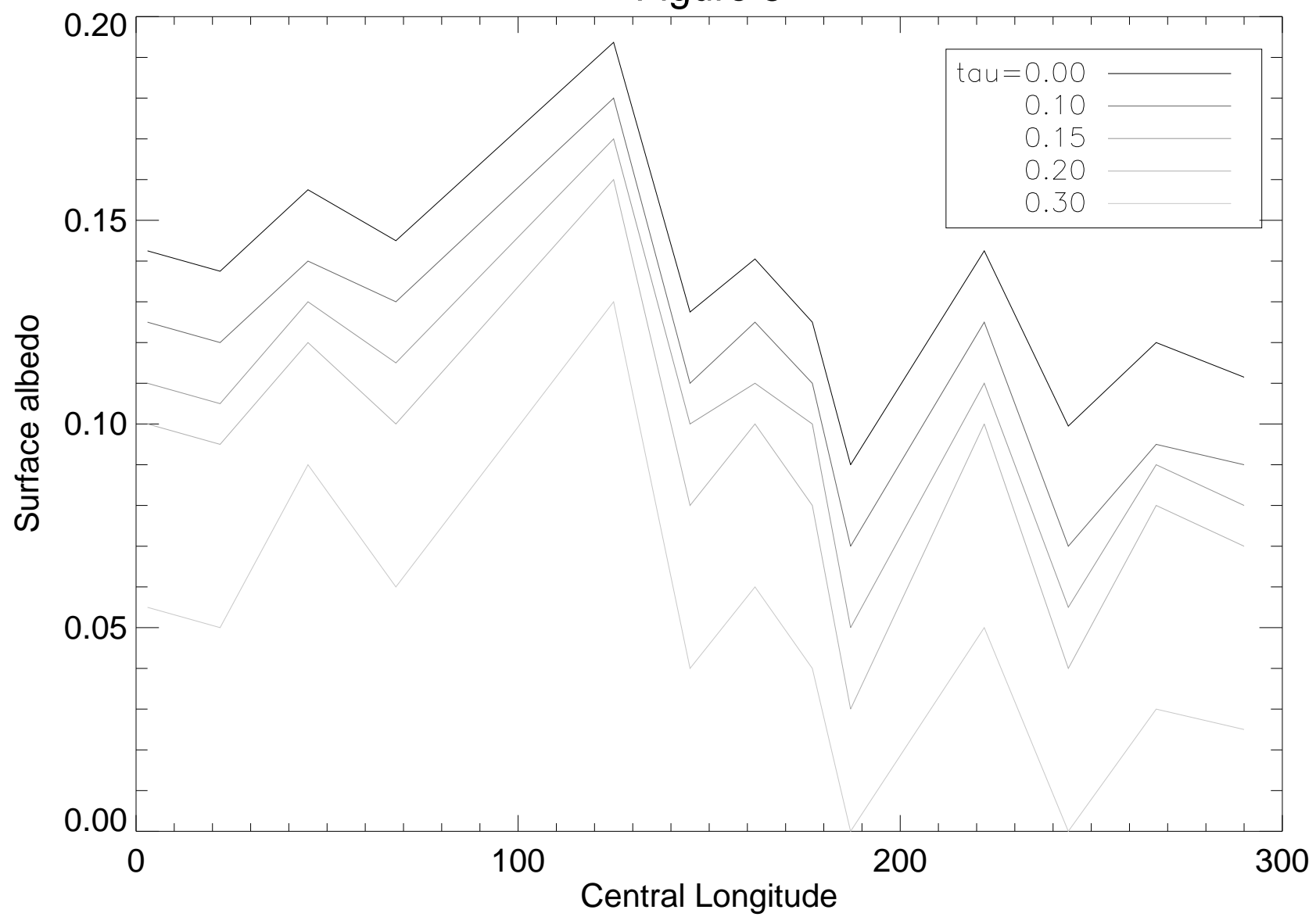


Figure 6

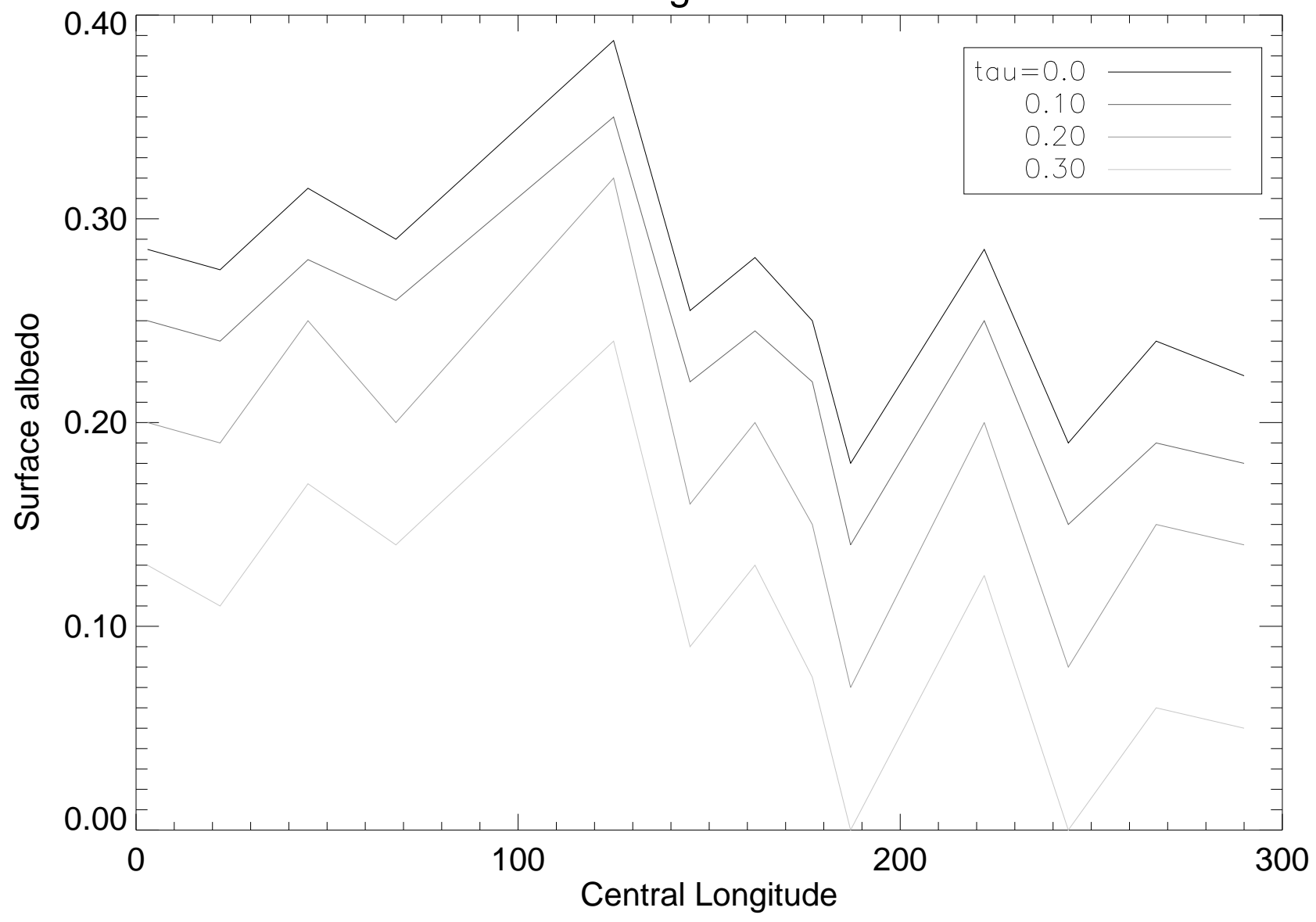

